\title{
Propagation of the Four-Wave Mixing signal in a molecular system: stochastic considerations of the thermal bath
}

\section{Propagación de la señal de Mezcla de cuatro ondas en un sistema molecular: Consideraciones estocásticas del baño térmico}

\author{
J. L. Paz ${ }^{(1,2, *}$, María Izquierdo(2), Luis G. Rodríguez(1) and César Costa Vera(1,3) \\ 1.Departamento de Física, Escuela Politécnica Nacional, Ladrón de Guevara, E11-253, 170517, Apdo. 17-12- \\ 866, Quito, Ecuador. \\ 2.Departamento de Química, Universidad Simón Bolívar, Apartado 89000, Caracas 1086, Venezuela. \\ 3.Grupo Ecuatoriano para el estudio Experimental y Teórico de Nanosistemas (GETNano), Diego de Robles y \\ Vía Interoceánica, USFQ, N104-E, Quito, Ecuador. \\ (*) E-mail: jlpaz@usb.ve \\ Received / Recibido: 12/05/2015 Accepted / Aceptado: 16/08/2015 \\ DOI: $10.7149 /$ OPA.48.3.225
}

\begin{abstract}
:
This work studies the spatial propagation of the Four-Wave Mixing (FWM) signal of a molecular system (malachite green) with two electronic levels immersed in a thermal bath, under a perturbative treatment at all order in the pump field and to first order in the probe and signal fields. Using the Optical Stochastic Bloch Equations OSBE, we present three approximations, two of them with analytical solutions and the third with a numerical approach, where the effects of the variation of pump intensity through the path optical are considered. We compare these results with the analogous in absence of the thermal reservoir. The stochastic effects induced by the solvent, due to the experimental conditions as relaxation times, chemical concentration of the solution and optical frequencies of the fields, diminishes the intensity responses compared with the same in absence of the bath.
\end{abstract}

Keywords: propagation, four-wave mixing, stochastic effects.

\section{RESUMEN:}

Este trabajo presenta un estudio detallado de la propagación espacial de la señal de mezcla de cuatro ondas (MCO) en un sistema molecular (malaquita verde) con dos niveles electrónicos inmerso en un baño térmico, bajo un tratamiento perturbativo a todos los órdenes en el campo de bombeo y primer orden en los campos de prueba y de señal. Usando las ecuaciones de Bloch ópticas estocásticas (EBOE), presentamos tres aproximaciones, dos de ellas son soluciones analíticas y la otra es de tipo numérico, donde se consideran los efectos de la variación de la intensidad del bombeo a través del camino óptico. Comparamos esos resultados con los análogos en ausencia del reservorio térmico. Los efectos estocásticos inducidos por el solvente, debido a las condiciones experimentales como tiempos de relajación, concentración química de la solución y frecuencias ópticas de los campos, disminuyen la respuesta de intensidad comparado con la misma en ausencia del baño.

Palabras clave: propagación, mezcla de cuatro ondas, efectos estocásticos. 


\section{REFERENCIAS Y ENLACES / REFERENCES AND LINKS}

[1] Y.R. Shen, The principles of nonlinear optics, John Wiley \& Sons, New Jersey, USA (2002)

[2] P.R. Berman, V.S. Malinovsky, Principles of laser spectroscopy and quantum optics, Princeton University Press, New Jersey, USA (2010).

[3] T. Passerat de Silans, C.S.L. Goncalves, D. Felinto, J.W.R. Tabosa, " Enhanced four-wave mixing via crossover resonance in cesium vapor," J. Opt. Soc. Am. B 28, 2220-2226 (2011) http://dx.doi.org/10.1364/JOSAB.28.002220

[4] W. Myron, I. Prigogine, Modern nonlinear optics, 2nd Ed. John Wiley \& Sons, Inc., Chicago, USA (2001)

[5] G.S. Agarwal, Selected papers on: fundamentals of quantum optics, SPIE Milestone Series, 103, (2006)

[6] S. Mukamel, R. Loring, "Nonlinear response function for time-domain and frequency domain fourwave mixing", J. Opt. Soc. Am.B 3, 595-606 (1986) http://dx.doi.org/10.1364/JOSAB.3.000595

[7] Moreno, M.; Vianna, S. Resonances in a three-level lambda system excited by an ultrashort pulse train, Latin America Optics and Photonics Conference Recife, Brazil (2010) http://dx.doi.org/10.1364/laop.2010.mb01

[8] M.S. Shahriar, P.R. Hemmer, "Generation of squeezed states and twin beams via non-degenerate fourwave mixing in a $\Lambda$ system", Opt. Commun. 158, 273-286 (1998). http://dx.doi.org/10.1016/S0030-4018(98)00528-8

[9] N. Heung-Ryoul, J. Wonho, "Analytic solutions of the optical Bloch equations", Opt. Commun. 283, 2353-2355 (2010) http://dx.doi.org/10.1016/j.optcom.2010.01.069

[10] R. Boyd, Nonlinear optics, 2nd. Ed. Academic press, New York, USA (2003)

[11] P.J. Colmenares, J. L. Paz, R. Almeida, "On the optical stochastic Bloch equations", Phys. Lett. A. 199, 163-168 (1995) http://dx.doi.org/10.1016/0375-9601(95)00081-D

[12] C. W. Gardiner, Handbook of Stochastic Methods for Physics, Chemistry and the Natural Sciences, Springer, Berlin (1985)

[13] R. Boyd, M.G. Raymer, P. Narum, D. Harter, "Four-wave parametric interactions in a strongly driven two-level system", Phys. Rev. A. 24, 411-423 (1981) http://dx.doi.org/10.1103/PhysRevA.24.411

[14] M. Gorayeb, J.L.Paz, A.J. Hernández, "Effect of inhomogeneous line broadening due to molecular collisions on a two-level system interacting with a high-intensity electromagnetic field", Opt. Commun. 251, 216-228 (2005) http://dx.doi.org/10.1016/i.optcom.2005.03.030

[15] A. Thorne, U. Litzén, S. Johansson, Spectrophysics: Principles and Applications, Springer-Verlag Berlin, Alemania (999)

[16] E. Hecht, Optics, Four edition, Pearson Addison Wesley, Boston, USA (2001)

[17] I. Reif, F. García-Golding, J.L.Paz, H.J. Franco, "Three levels of approximation in the study of the propagation of the Rayleigh-type optical mixing signal", J. Opt. Soc. Am. B. 8, 2470-2476 (1991) http://dx.doi.org/10.1364/JOSAB.8.002470

[18] Y. Wenjie, Y. Zhongyuan, L. Yumin, J. Boyong, "Linear and nonlinear optical absorption coefficients and refractive index changes in strained GaN/AlN quantum dots", Physica E, 41, 1382-1385 (2009) http://dx.doi.org/10.1016/.physe.2009.03.003

[19] J.L.Paz, A.Mendoza-García, "Solvent influence on the nonlinear optical properties of molecularsystems in the presence of degenerate and non-degenerate four-wave mixing", J. Mod. Opt. 59, 71-82 (2012) http://dx.doi.org/10.1080/09500340.2011.639962

[20] J. Reimers, "Computational methods for large systems: electronic structure approaches for biotechnology and nanotechnology", John Wiley \& Sons Inc., Chicago, USA (2011) http://dx.doi.org/10.1002/9780470930779 
[21] J.L.Paz, A. Mastrodomenico, César costa-Vera, Jaime Cárdenas-García and Luis G. Rodríguez, "Rotating wave approximation effects on the nonlinear optical responses of complex molecular systems using a four-wave mixing signal", J. Mod. Opt. 62,403-411 (2015) http://dx.doi.org/10.1080/09500340.2014.986235

[22] N.G.Van Kampen, Stochastic Processes in Physics, North-Holland, New York, USA (1981)

[23] R. Boyd, Nonlinear Optics, Academic Press Inc., California (1992)

[24] A. Marcano O., F. García-Golding, "Power effects in polarization spectroscopy on a water solution of malachite green", J. Chem. Phys. 82, 1242-1245 (1985) http://dx.doi.org/10.1063/1.448444

[25] J. L. Paz, R. Almeida, P.J. Colmenares, "A formalism to analyze effects of the electromagnetic field on the optical properties of a two-level system immersed in a thermal bath", Phys. Lett. A., 226, 365-374 (1997) http://dx.doi.org/10.1016/S0375-9601(96)00923-1

\section{Introducción}

La interacción de la materia con un campo eléctrico incidente de alta intensidad se ha estudiado teórica y experimentalmente en diversos procesos ópticos no lineales [1]. Estas interacciones se dicen "no lineales" cuando la respuesta de un sistema frente a un campo aplicado depende de manera no lineal respecto a la intensidad del mismo. El origen de estas interacciones está sujeto a un modelo simple basado en las propiedades del material y en la forma en la que éstas se propagan en el medio [2-5]. Existen diversos procesos multi-fotónicos, entre los cuales resaltan: la generación de segundos y terceros armónicos, la generación de una suma o diferencia de frecuencias, y los procesos de polarización a tercer orden, principalmente la llamada mezcla de cuatro ondas (MCO) [6]. Estos últimos ocurren cuando al menos, dos longitudes de onda distintas (un campo de alta intensidad conocido como haz de bombeo y un campo de menor intensidad conocido como haz de prueba) pasan a través del recorrido óptico, generando productos espectrales o mezclas de ondas. La cuarta onda es generada por la polarización inducida en el medio por los campos aplicados cuya frecuencia puede escribirse como combinación lineal de las frecuencias de los campos incidentes. Los procesos de MCO en medios moleculares se han estudiado en diversas configuraciones, desde sistemas de dos niveles electrónicos [7], hasta sistemas de tres y cuatro niveles tipo $\Lambda$ y doble $\Lambda$, respectivamente [8]. Sin embargo, el sistema molecular más estudiado es un sistema de dos niveles, dado que los estados enlazantes de muchas moléculas pueden describirse a través de este par de estados, y donde las ecuaciones de movimiento son bien conocidas. Teóricamente, los fenómenos no lineales se estudian en un esquema perturbativo bajo el formalismo de la matriz densidad, y la ecuación de Liouville para su descripción dinámica. Al considerar los términos de relajación asociados al proceso, se derivan las ecuaciones de Bloch ópticas convencionales (EBOC), análogas en su forma a las ecuaciones magnéticas de Bloch [9]. A través de las poblaciones y coherencias inherentes a las EBOC se estudia la dinámica del sistema expuesto a la radiación de alta intensidad. En este contexto, una interacción fuerte del campo con un medio no homogéneo puede inducir múltiples colisiones, por lo que la naturaleza microscópica del sistema puede cambiar de manera importante. Para resolver este problema algo más complicado, proponemos una metodología basada en una descripción estocástica, donde la interacción soluto-solvente induce un corrimiento aleatorio en la frecuencia de resonancia del sistema de dos niveles o frecuencia de Bohr $\omega_{0}$. Esto genera así un ensanchamiento del nivel superior, lo cual transforma el parámetro usual $\omega_{0}$ en una variable estocástica $\xi(t)$, definida como $\xi(t)=\omega_{0}+\varepsilon(t)$ y las EBOC en ecuaciones de Bloch ópticas estocásticas EBOE [10,11]. Es claro que con este tratamiento la promediación en el conjunto estadístico sobre todas las realizaciones de la variable aleatoria $\langle\xi(t)\rangle$ conduce a $\omega_{0}$ [12]. En el contexto de un ensanchamiento inhomogéneo, el conjunto estadístico correspondiente es un conjunto de sistemas de dos niveles donde cada uno es caracterizado por una frecuencia $\omega_{0}$. En tal sentido, el camino de cálculo que se propone en este trabajo, consiste en derivar de las EBOE, las componentes de Fourier asociadas tanto a las coherencias como a las poblaciones que oscilan a las frecuencias de interés, y con ello, la polarización inducida a la frecuencia de los campos de bombeo, prueba y señal de MCO. Empleando esta polarización inducida como fuente en las ecuaciones de 
Maxwell en un medio no magnético, obtenemos expresiones de propagación de las amplitudes de cada campo, como función tanto de la absorción dentro del material como de los parámetros de acoplamiento entre los campos participantes. Con estas ecuaciones de propagación, es posible estudiar el comportamiento de la señal de MCO a lo largo del camino óptico, como función de las distintas formas de absorción del haz de intensidad fuerte. Aunque en la literatura se han reportado estudios de propagación con diversas metodologías de cálculo, considerando inclusive los efectos del solvente [13-17], la mayoría de las ecuaciones asociadas a procesos multi-fotónicos no se han resuelto analíticamente. Por tanto en este trabajo se estudia detalladamente las modificaciones que tiene la evaluación de los promedios estocásticos que surgen sobre las formas de propagación de la señal de MCO. También se comparan aquí las distintas formas de propagación en ausencia y presencia del efecto del solvente, los cuales se parametrizan con la concentración de la solución química del soluto, los tiempos de relajación naturales de los colorantes empleados, la frecuencia óptica de los campos incidentes, momentos dipolares inducidos, así como de las variables propias del ruido que distingue al solvente [18-20].

\section{Ecuaciones de Bloch ópticas estocásticas. Componentes de Fourier}

Tomando como punto de partida la ecuación de Liouville-Von Newmann para la matriz densidad, y considerando un sistema de dos niveles moleculares $|a\rangle$ y $|\mathrm{b}\rangle$ interactuando con campos electromagnéticos clásicos en la aproximación tipo dipolo-eléctrico e inmerso en un baño térmico cuya presencia se manifiesta en el corrimiento de la frecuencia natural de resonancia $\omega_{0}$ a través de la variable estocástica $\xi(t)$, podemos expresar la dinámica temporal de nuestro sistema bajo la forma de las EBOE, definidas como:

$$
\begin{aligned}
& \dot{\rho}(\mathrm{t})=\mathrm{A}_{\xi}(\mathrm{t})+\mathbb{R} \\
& \rho(\mathrm{t})=\left(\begin{array}{c}
\rho_{\mathrm{ba}} \\
\rho_{\mathrm{ab}} \\
\rho_{\mathrm{D}}
\end{array}\right) ; \quad \mathrm{A}_{\xi}(\mathrm{t})=\left(\begin{array}{ccc}
-\xi_{\mathrm{t}} & 0 & \mathrm{i} \Omega \\
0 & -\xi_{\mathrm{t}}^{*} & -\mathrm{i} \Omega^{*} \\
2 \mathrm{i} \Omega^{*} & -2 \mathrm{i} \Omega & -1 / \mathrm{T}_{1}
\end{array}\right) ;=\left(\begin{array}{c}
0 \\
0 \\
(0) / \mathrm{T}_{1}
\end{array}\right)
\end{aligned}
$$

donde $\xi_{\mathrm{t}}=\mathrm{i} \xi(\mathrm{t})+1 / \mathrm{T}_{2} ; \mathrm{A}_{\xi}(\mathrm{t})$ es la matriz radiativa que incluye la frecuencia estocástica dependiente del tiempo, y donde -, se corresponde con la matriz de relajación, que comprende los valores de equilibrio de las poblaciones controladas por el tiempo de relajación longitudinal $\mathrm{T}_{1}$. Considerando las aproximaciones de la onda rotante (AOR) y de estado estacionario, podemos obtener las siguientes relaciones para las componentes de Fourier de las coherencias $\rho_{\mathrm{ba}}\left(\omega_{\mathrm{j}}\right)$ y de la diferencia de poblaciones $\rho_{\mathrm{D}}(\mathrm{n} \Delta)$, las cuales oscilan a las frecuencias de interés $\omega_{\mathrm{j}} \mathrm{y} \mathrm{n} \Delta$, respectivamente:

$$
\begin{aligned}
& \left.\mathrm{D}_{2 \mathrm{n}+1 \text { ba }}\left({ }_{1}+\mathrm{n}\right)=\mathrm{i} \quad{ }_{1} \mathrm{D}^{(\mathrm{n})}\right)+\mathrm{i}_{2}{ }_{\mathrm{D}}[(\mathrm{n}+1)]+\mathrm{i}_{3 \mathrm{D}}\left[\left(\begin{array}{ll}
\mathrm{n} & 1
\end{array}\right)\right]
\end{aligned}
$$

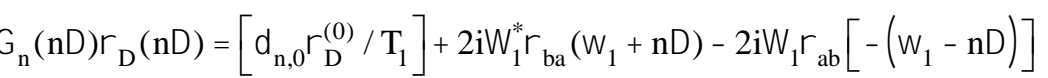

$$
\begin{aligned}
& +2 \mathrm{i}_{2}^{*} \text { ba }\left[1+\left(\begin{array}{ll}
n & 1
\end{array}\right)\right] 2 \mathrm{i}_{2} \text { ab }\left\{\left[\begin{array}{ll}
1 & (\mathrm{n}+1)
\end{array}\right]\right\} \\
& \left.+2 \mathrm{i}_{3 \text { ba }}^{*}[1+(\mathrm{n}+1)] 2 \mathrm{i}_{3 \text { ab }}\left\{\left[\begin{array}{lll}
1 & (\mathrm{n} & 1
\end{array}\right)\right]\right\} \\
& \mathrm{D}_{2 \mathrm{n}+1}=\frac{1}{\mathrm{~T}_{2}}+\mathrm{i}\left[\xi-\left(\omega_{1}+\mathrm{n} \Delta\right)\right] \text { y } \Gamma_{\mathrm{n}}(\mathrm{n} \Delta)=\frac{1}{\mathrm{~T}_{1}}-\mathrm{in} \Delta, \quad \mathrm{n}=1,2,3 \ldots
\end{aligned}
$$

El término $\mathrm{D}_{2 \mathrm{n}+1}$ está asociado a las componentes resonantes (AOR), para valores de $\mathrm{n}=0,1,-1$ para los haces de bombeo, señal de MCO y prueba, respectivamente. Además, definimos $D_{1} D_{2}$, con $\Omega=\vec{\mu}_{\mathrm{ba}} \cdot \overrightarrow{\mathrm{E}}(\mathrm{t}) / \hbar$ como la frecuencia de Rabí asociado al campo $\overrightarrow{\mathrm{E}}$, la cual define el parámetro de intensidad del acoplamiento radiación-materia; $T_{2}$ expresa el tiempo de relajación transversal asociado a la pérdida de coherencia inducida; $\quad={ }_{1}{ }_{2}$ es la desintonización de los campos incidentes de frecuencias $\omega_{1} \mathrm{y}$ 
$\omega_{2}$. En este punto es conveniente mencionar que la expansión considerada es válida en el caso que los elementos de la matriz densidad sean funciones estocásticas estacionarias, esto es, con funciones de correlación propias y valores medios definidos. Nuestro modelo se restringe a un desarrollo perturbativo a primer orden en las amplitudes de la prueba y de señal de MCO, mientras que el haz de bombeo es considerado a todos los órdenes, sin restricción alguna. Nuestro tratamiento no incluye los procesos de relajación espontánea. Dado que permanecemos en la AOR, no consideramos explícitamente los momentos dipolares permanentes de la molécula bajo estudio (colorante orgánico malaquita verde), porque estos sólo tienen efecto al considerar procesos anti-resonantes al margen de la AOR [21]. Bajo estas consideraciones, tenemos entonces, el siguiente conjunto de ecuaciones acopladas para las componentes de Fourier de las coherencias y diferencias de poblaciones a las frecuencias de interés:

$$
\begin{aligned}
& \mathrm{D}_{3 \text { ba }}\left({ }_{3}\right)=\mathrm{i}_{1} \mathrm{D}_{\mathrm{D}}()+\mathrm{i}_{3} \underset{\mathrm{D}}{\mathrm{dc}} \\
& \Gamma_{1}(\Delta) \rho_{\mathrm{D}}(\Delta)=2 \mathrm{i} \Omega_{1}^{*} \rho_{\mathrm{ba}}\left(\omega_{3}\right)+2 \mathrm{i} \Omega_{2}^{*} \rho_{\mathrm{ba}}\left(\omega_{1}\right)-2 \mathrm{i} \Omega_{1} \rho_{\mathrm{ab}}\left(-\omega_{2}\right)-2 \mathrm{i} \Omega_{3} \rho_{\mathrm{ab}}\left(-\omega_{1}\right) \\
& \mathrm{D}_{1} \rho_{\mathrm{ba}}\left(\omega_{1}\right)=\mathrm{i}_{1} \rho_{\mathrm{D}}^{\mathrm{dc}} ; \quad \mathrm{D}_{1}^{*} \rho_{\mathrm{ab}}\left(-\omega_{1}\right)=-\mathrm{i} \Omega_{1}^{*} \rho_{\mathrm{D}}^{\mathrm{dc}} \\
& \mathrm{D}_{2}^{*} \rho_{\mathrm{ab}}\left(-\omega_{2}\right)=-\mathrm{i} \Omega_{1}^{*} \rho_{\mathrm{D}}(\Delta)-\mathrm{i} \Omega_{2}^{*} \rho_{\mathrm{D}}^{\mathrm{dc}}
\end{aligned}
$$

El término $\rho_{\mathrm{D}}^{\mathrm{dc}}$ define el término de corriente directa asociado a la componente de Fourier que oscila a cero frecuencia, el cual es una medida de cuanto se alejan las poblaciones de la distribución canónica de Boltzmann en presencia del campo intenso de bombeo. Desacoplando el conjunto de ecuaciones (4-7) nos queda:

$$
\begin{aligned}
& \rho_{\mathrm{ba}}\left(\omega_{1}\right)=\left[\Phi^{(\mathrm{incoh})}\left(\omega_{1}\right)\right] \Omega_{1} \\
& \rho_{\mathrm{ba}}\left(\omega_{2}\right)=\left[\Phi^{(\mathrm{incoh})}\left(\omega_{2}\right)+\Phi^{(\mathrm{coh})}\left(\omega_{2}\right)\right] \Omega_{2}+\Phi^{(\mathrm{acop})}\left(\omega_{2}\right) \Omega_{1}^{2} \Omega_{3}^{*} \\
& \rho_{\mathrm{ba}}\left(\omega_{3}\right)=\left[\Phi^{(\mathrm{incoh})}\left(\omega_{3}\right)+\Phi^{(\mathrm{coh})}\left(\omega_{3}\right)\right] \Omega_{3}+\Phi^{(\mathrm{acop})}\left(\omega_{3}\right) \Omega_{1}^{2} \Omega_{2}^{*} \\
& \text { (incoh) } \left._{(}{ }_{k}\right)=\frac{i \frac{d c}{D}}{D_{k}}, k=1,2,3 \text {. } \\
& { }^{(\mathrm{coh})}\left({ }_{2}\right)=\left(\frac{2 \mathrm{i}\left|{ }_{1}\right|^{2}}{\mathrm{D}_{2}{ }^{*}} \mathrm{f}_{2,1}\right) \mathrm{dc}_{\mathrm{D}}^{\mathrm{dc}} ; \quad{ }^{(\mathrm{coh})}\left({ }_{3}\right)=\left(\frac{\left.2 \mathrm{i}||_{1}\right|^{2}}{\mathrm{D}_{3} \mathrm{f}_{3,1}}\right) \frac{\mathrm{dc}}{\mathrm{D}} \\
& \text { (acop) }\left({ }_{2}\right)=\left(\frac{2 \mathrm{i}}{\mathrm{D}_{2} \mathrm{f}_{1}} \mathrm{f}_{1,3}\right) \underset{\mathrm{D}}{\mathrm{dc}} ; \quad \quad\left(\text { acop) }\left({ }_{3}\right)=\left(\frac{2 \mathrm{i}}{\mathrm{D}_{3} \mathrm{f}_{1,2}}\right) \frac{\mathrm{dc}}{\mathrm{D}}\right. \\
& \mathrm{f}_{\mathrm{n},-\mathrm{m}}=\frac{1}{D_{\mathrm{n}}}+\frac{1}{D_{m}^{*}} .
\end{aligned}
$$

La forma como hemos definido los términos que aparecen en las distintas componentes de Fourier de las coherencias, nos permite asociar la contribución "incoherente" con la reducción en la población relativa de los dos estados debido a los efectos saturativos del haz de bombeo, relacionado con el mecanismo de absorción en el material; la componente "coherente", a su vez, señala la interferencia entre los haces débiles y fuertes, debido a la oscilación de poblaciones oscilante a la frecuencia de desintonización $\Delta$. El último término en las ec. (9), indica el proceso de "acoplamiento" de los haces de bombeo y prueba que forman una red de difracción que permite la dispersión del bombeo generando fotones a la frecuencia $\omega_{3}$, mientras que en la ec.(10) este término señala la dispersión del bombeo con la red de oscilación bombeoseñal que permite la generación de fotones a la frecuencia $\omega_{2}$. La asimetría de las ecs. (9) y (10) con relación a la ec.(8), se debe a la forma asimétrica del tratamiento perturbativo para los haces considerados. Dada la baja intensidad de los haces de prueba y señal, no tenemos mecanismos de 
acoplamiento que permite la generación de fotones a la frecuencia $\omega_{1}$ del haz fuerte. Para la componente a cero-frecuencia $\rho_{\mathrm{D}}^{\mathrm{dc}}$, tenemos:

$$
\rho_{\mathrm{D}}^{\mathrm{dc}}=\frac{\left|\mathrm{D}_{1}\right|^{2} \mathrm{~T}_{2}^{2}}{\left|\mathrm{D}_{1}\right|^{2} \mathrm{~T}_{2}^{2}+4 \mathrm{~S}} \rho_{\mathrm{D}}^{(0)}
$$

Aquí, $\mathrm{S}=\left|\Omega_{1}\right|^{2} \mathrm{~T}_{1} \mathrm{~T}_{2}$ se define como el parámetro de saturación. Es importante señalar, que la dependencia con la variable estocástica de las componentes $\rho_{\mathrm{ba}}\left(\omega_{\mathrm{j}}\right)$ es a través de los términos resonantes $D_{2 n+1}$. Aquí es importante mencionar, que dado que las coherencias son funciones estocásticas, es necesario tomar el promedio sobre todas las realizaciones de la variable $\xi(\mathrm{t})$ en el conjunto estadístico. Sobre este particular, seguimos un procedimiento distinto al propuesto por Van Kampen [22] y resolvemos las ecuaciones como si ellas fueran deterministas y, reconociendo a continuación la dependencia con la variable aleatoria, tomamos el promedio de conjunto sobre la distribución de estados con frecuencias moleculares entre and $+\mathrm{d}$. Por tanto, la solución en esta aproximación ergódica de las ecuaciones estocásticas, resulta en que el promedio de conjunto sustituye el promedio temporal, con lo cual se evita la situación de tener que resolver promedios que involucran ruidos multiplicativos. Consecuentemente, tomando el promedio en las ecs. (8-10) de las coherencias sobre la distribución de la variable estocástica $\xi(\mathrm{t})$ y usando la aproximación escalar [23] tenemos:

$$
\begin{aligned}
\left\langle\rho_{\mathrm{ba}}\left(\omega_{1}\right)\right\rangle_{\xi}= & \frac{\mathrm{i} \mu_{\mathrm{ba}}}{\hbar}\left\langle\frac{\rho_{\mathrm{D}}^{\mathrm{dc}}}{\mathrm{D}_{1}}\right\rangle_{\xi} \mathrm{E}_{1}\left(\omega_{1}\right) \\
\left\langle\rho_{\mathrm{ba}}\left(\omega_{2}\right)\right\rangle_{\xi} & =\frac{i \mu_{\mathrm{ba}}}{\hbar}\left\langle\frac{\rho_{\mathrm{D}}^{\mathrm{dc}}}{\mathrm{D}_{2}}\right\rangle_{\xi} \mathrm{E}_{2}\left(\omega_{2}\right)-\frac{2 \mathrm{i}\left|\mu_{\mathrm{ba}}\right|^{2} \mu_{\mathrm{ab}}}{\hbar^{3}}\left\langle\frac{\rho_{\mathrm{D}}^{\mathrm{dc}}}{\mathrm{D}_{2} \Psi_{1}^{*}} \mathrm{f}_{2,-1}\right\rangle_{\xi} \mathrm{E}_{1}\left(\omega_{1}\right) \mathrm{E}_{1}^{*}\left(-\omega_{1}\right) \mathrm{E}_{2}\left(\omega_{2}\right) \\
& -\frac{2 \mathrm{i}\left|\mu_{\mathrm{ba}}\right|^{2} \mu_{\mathrm{ab}}}{\hbar^{3}}\left\langle\frac{\rho_{\mathrm{D}}^{\mathrm{dc}}}{\mathrm{D}_{2} \Psi_{1}^{*}} \mathrm{f}_{1,-3}\right\rangle_{\xi} \mathrm{E}_{1}\left(\omega_{1}\right) \mathrm{E}_{1}\left(\omega_{1}\right) \mathrm{E}_{3}^{*}\left(-\omega_{3}\right) \\
\left\langle\rho_{\mathrm{ba}}\left(\omega_{3}\right)\right\rangle_{\xi} & =\frac{\left.\mathrm{i} \mu_{\mathrm{ba}} / \frac{\rho_{\mathrm{D}}^{\mathrm{dc}}}{\hbar}\right\rangle_{3}}{\mathrm{D}_{3}} \mathrm{E}_{3}\left(\omega_{3}\right)-\frac{2 \mathrm{i}\left|\mu_{\mathrm{ba}}\right|^{2} \mu_{\mathrm{ab}}}{\hbar^{3}}\left\langle\frac{\rho_{\mathrm{D}}^{\mathrm{dc}}}{\mathrm{D}_{3} \Psi_{1}} \mathrm{f}_{3,-1}\right\rangle_{\xi} \mathrm{E}_{1}\left(\omega_{1}\right) \mathrm{E}_{1}^{*}\left(-\omega_{1}\right) \mathrm{E}_{3}\left(\omega_{3}\right) \\
& -\frac{2 \mathrm{i}\left|\mu_{\mathrm{ba}}\right|^{2} \mu_{\mathrm{ab}}}{\hbar^{3}}\left\langle\frac{\rho_{\mathrm{D}}^{\mathrm{dc}}}{\mathrm{D}_{2} \Psi_{1}} \mathrm{f}_{1,-2}\right\rangle_{\xi} \mathrm{E}_{1}\left(\omega_{1}\right) \mathrm{E}_{1}\left(\omega_{1}\right) \mathrm{E}_{2}^{*}\left(-\omega_{2}\right)
\end{aligned}
$$

donde ${ }_{1}={ }_{1}(\quad)+2\left|{ }_{1}\right|^{2} \mathrm{f}_{3,2}$.

La componente de Fourier de la polarización local no lineal inducida para cada una de las frecuencias de los campos presentes, está dada por:

$$
\mathrm{P}\left({ }_{1}+\mathrm{n}\right)=\mathrm{N}\left\langle\left\langle{ }_{\text {ba }}\left({ }_{1}+\mathrm{n}\right)\right\rangle_{\left({ }_{0}\right)} \text { ab }\right\rangle
$$

siendo $\mathrm{N}$ la concentración de moléculas del soluto activo a la radiación. Los promedios externos denotan un promedio sobre todas las posibles orientaciones moleculares del sistema de dos niveles, mientras que el promedio interno recae sobre la distribución de frecuencias moleculares $v\left(\omega_{0}\right)=\mathrm{L}\left(\omega_{0}\right), \mathrm{g}\left(\omega_{0}\right)$. De esta forma y con las consideraciones arriba señaladas, tenemos que:

$$
\begin{aligned}
& \mathrm{P}\left({ }_{1}\right)={ }^{\mathrm{SV}}\left({ }_{1}\right) \mathrm{E}_{1}\left({ }_{1}\right)+{ }^{\text {(incoh) }}\left({ }_{1}\right) \mathrm{E}_{1}\left({ }_{1}\right) \\
& \mathrm{P}\left(\omega_{2}\right)=\chi^{\mathrm{SV}}\left(\omega_{2}\right) \mathrm{E}_{2}\left(\omega_{2}\right)+\left\{\chi^{(\text {incoh })}\left(\omega_{2}\right)+\tilde{\chi}^{(\mathrm{coh})}\left(\omega_{2}\right)\right\} \mathrm{E}_{2}\left(\omega_{2}\right)+\chi^{(\mathrm{acop})}\left(\omega_{2}\right) \mathrm{E}_{1}\left(\omega_{1}\right) \mathrm{E}_{1}\left(\omega_{1}\right) \mathrm{E}_{3}^{*}\left(-\omega_{3}\right),
\end{aligned}
$$




$$
\mathrm{P}\left(\omega_{3}\right)=\chi^{\mathrm{SV}}\left(\omega_{3}\right) \mathrm{E}_{3}(\omega)+\left\{\chi^{(\mathrm{incoh})}\left(\omega_{3}\right)+\tilde{\chi}^{(\mathrm{coh})}\left(\omega_{3}\right)\right\} \mathrm{E}_{3}\left(\omega_{3}\right)+\chi^{(\mathrm{acop})}\left(\omega_{3}\right) \mathrm{E}_{1}\left(\omega_{1}\right) \mathrm{E}_{1}\left(\omega_{1}\right) \mathrm{E}_{2}^{*}\left(-\omega_{2}\right)
$$

En este caso, la componente incoherente, toma la misma forma independientemente de que campo electromagnético se esté considerando.

$$
\chi^{(\text {incoh })}\left(\omega_{\mathrm{k}}\right)=\frac{\mathrm{i}\left|\mu_{\mathrm{ba}}\right|^{2} \mathrm{~N}}{\hbar}\left\langle\frac{\rho_{\mathrm{D}}^{\mathrm{dc}}}{\mathrm{D}_{\mathrm{k}}}\right\rangle_{\xi} \quad \mathrm{k}=1,2,3 .
$$

A su vez, la componente coherente efectiva $\tilde{\chi}^{(\mathrm{coh})}\left(\omega_{\mathrm{k}}\right)$ asociada a los campos de prueba y de señal, viene dada por las expresiones siguientes:

$$
\begin{aligned}
& \tilde{\chi}^{(\mathrm{coh})}\left(\omega_{2}\right)=-\frac{2 \mathrm{i}\left|\mu_{\mathrm{ba}}\right|^{4} \mathrm{~N}}{\hbar^{3}}\left\langle\frac{\rho_{\mathrm{D}}^{\mathrm{dc}}}{\mathrm{D}_{2} \Psi_{1}^{*}} \mathrm{f}_{2,-1}\right\rangle_{\xi}\left|\mathrm{E}_{1}\left(\omega_{1}\right)\right|^{2} \\
& \tilde{\chi}^{(\mathrm{coh})}\left(\omega_{3}\right)=-\frac{2 \mathrm{i}\left|\mu_{\mathrm{ba}}\right|^{4} \mathrm{~N}}{\hbar^{3}}\left\langle\frac{\rho_{\mathrm{D}}^{\mathrm{dc}}}{\mathrm{D}_{3} \Psi_{1}} \mathrm{f}_{3,-1}\right\rangle_{\xi}\left|\mathrm{E}_{1}\left(\omega_{1}\right)\right|^{2}
\end{aligned}
$$

Finalmente, las componentes de acoplamiento de las susceptibilidades, vienen expresadas de la forma:

$$
\begin{aligned}
& \chi^{(\text {acop })}\left(\omega_{2}\right)=-\frac{2 i\left|\mu_{b a}\right|^{4} N}{\hbar^{3}}\left\langle\frac{\rho_{D}^{d c}}{D_{2} \Psi_{1}^{*}} f_{1,-3}\right\rangle_{\xi} \\
& \chi^{(\text {acop })}\left(\omega_{3}\right)=-\frac{2 i\left|\mu_{b a}\right|^{4} N}{\hbar^{3}}\left\langle\frac{\rho_{D}^{d c}}{D_{3} \Psi_{1}} f_{1,-2}\right\rangle_{\xi}
\end{aligned}
$$

Es necesario hacer notar que en la definición de la susceptibilidad coherente (coh) de los haces de prueba y de señal, está contenida explícitamente la intensidad del campo de bombeo, a diferencia de las incoherentes (incoh) o de acoplamientos (acop) que siguen estrictamente la definición tensorial de la forma de polarización y donde la dependencia con la intensidad del haz de bombeo es de forma implícita, a través del parámetro de saturación. Tal como indicamos anteriormente, los promedios en el conjunto estadístico sobre la variable aleatoria $\xi(\mathrm{t})$ están contenidos estrictamente tanto en $D_{\xi}$ como en $\mathrm{dc}^{\mathrm{D}}$. Hemos incorporado ${ }^{\mathrm{Sv}}\left({ }_{\mathrm{k}}\right)$ en cada una de las componentes de polarización para incluir la presencia al solvente. Dado que necesitamos en cada uno de los casos, evaluar el promedio del conjunto estadístico conformado por sistema de dos niveles, se considerará por simplicidad la condición $\omega_{1} \approx \omega_{2}$ en el cálculo. Esta situación se define comúnmente como la condición relativa asociada al máximo del efecto de las pulsaciones de poblaciones [24]. Esta condición convierte todas las resonancias $D_{1}=D_{2}=D_{3}$ en $D_{\xi}$. Considerando la condición indicada, y suponiendo que la frecuencia óptica $\omega_{1} \approx\langle\xi(t)\rangle=\omega_{0}$ (dado el teorema de límite central [12,23]) podemos demostrar que $\Psi_{1} \approx \mathrm{T}_{1}^{-1}$ lo cual se sustenta en el hecho que para los colorantes orgánicos típicos, se cumple que $\mathrm{X}=4 \mathrm{~S} /|\mathrm{D}|^{2} \mathrm{~T}_{2}^{2} \quad$ 1. Por lo tanto, la componente "dc" se puede expresar de la forma: $\rho_{\mathrm{D}}^{\mathrm{dc}}=(1+\mathrm{X})^{-1}=1-\mathrm{X}+\mathrm{X}^{2}-\mathrm{X}^{3}+\ldots$ A partir de esto, y luego de algunas manipulaciones algebraicas, encontramos que las Susceptibilidades incoherentes (bombeo, prueba y señal de MCO) cumplen:

$$
\begin{aligned}
& { }^{\text {incoh) }}\left({ }_{j}\right)=C_{1}^{M}{ }_{k=0}^{M}\left\langle A^{(k)}\right\rangle, j=1,2,3 \\
& A^{(k)}=\frac{1}{D|D|^{2 k}} ; C_{1}=\frac{i\left|\mu_{b a}\right|^{2} N \rho_{D}^{(0)}}{\hbar} ; \quad k=(1)^{k}\left(\frac{4 S}{T_{2}^{2}}\right)^{k} .
\end{aligned}
$$


En todos los casos se requiere hasta un cierto valor $\mathrm{M}$ del índice de suma $\mathrm{k}$ para asegurar la convergencia de esta expresión. Para las susceptibilidades efectivas coherentes, tenemos la siguiente expresión:

$$
\begin{aligned}
& \tilde{\chi}^{(\mathrm{coh})}\left(\omega_{\mathrm{m}}\right)=\mathrm{C}_{2} \sum_{\mathrm{k}=0}^{\mathrm{M}} \lambda_{\mathrm{k}}\left\langle\mathrm{B}^{(\mathrm{k})}\right\rangle_{\xi}\left|\mathrm{E}_{1}\left(\omega_{1}\right)\right|^{2}, \mathrm{~m}=2,3 \\
& \mathrm{~B}^{(\mathrm{k})}=\frac{1}{\mathrm{D}_{\xi}\left|\mathrm{D}_{\xi}\right|^{2(\mathrm{k}+1)}} \text { y } \mathrm{C}_{2}=-\frac{2 \mathrm{i}\left|\mu_{\mathrm{ba}}\right|^{4} \mathrm{~N}}{\hbar^{3}}\left(\frac{2 \mathrm{~T}_{1}}{\mathrm{~T}_{2}}\right) \rho_{\mathrm{D}}^{(0)},
\end{aligned}
$$

donde es posible demostrar que se cumple la relación: $\left\langle B^{(n)}\right\rangle_{\xi}=\left\langle A^{(n+1)}\right\rangle_{\xi}$.

Finalmente, las susceptibilidades producto de los acoplamientos están dadas por:

$$
\chi^{(\text {acop })}\left(\omega_{\mathrm{m}}\right)=\mathrm{C}_{2} \sum_{\mathrm{k}=0}^{\mathrm{M}} \lambda_{\mathrm{k}}\left\langle\mathrm{B}^{(\mathrm{k})}\right\rangle_{\xi}, \mathrm{m}=2,3 .
$$

Como se puede observar, todas las susceptibilidades consideradas, están asociadas estrictamente al conocimiento del término $\left\langle\mathrm{A}^{(\mathrm{k})}\right\rangle_{\xi}$ de forma relativa a las susceptibilidades incoherentes. En este trabajo suponemos perfiles espectrales lorentzianos dado que el ensanchamiento de las líneas se debe principalmente a colisiones moleculares en el sistema soluto-solvente, es decir

$$
\mathrm{L}(\xi)=\frac{1}{\pi}\left(\frac{\sigma_{\mathrm{L}}}{\sigma_{\mathrm{L}}^{2}+\left(\xi-\omega_{0}\right)^{2}}\right)
$$

donde $\sigma_{\mathrm{L}}$ es la varianza de la distribución. Para resolver los promedios anteriores, usamos el esquema siguiente [25], separando en partes real e imaginaria el término de orden cero:

$$
\begin{aligned}
& \operatorname{Re}\left\langle\mathrm{A}^{(0)}\right\rangle=\frac{\mathrm{L}}{\mathrm{T}_{2}} \int_{\infty}^{\infty}\left[\mathrm{x}^{2}+\begin{array}{l}
2 \\
\mathrm{~L}
\end{array}\right]^{1}\left[\left(1 / \mathrm{T}_{2}\right)^{2}+\left(\begin{array}{ll}
1 & \mathrm{x})^{2}
\end{array}\right]^{1} \mathrm{dx},\right. \\
& \operatorname{Im}\left\langle\mathrm{A}^{(0)}\right\rangle_{\xi}=-\frac{\sigma_{\mathrm{L}}}{\mathrm{T}_{2} \pi} \int_{-\infty}^{\infty}\left[\mathrm{x}^{2}+\sigma_{\mathrm{L}}^{2}\right]^{-1}\left[\left(1 / \mathrm{T}_{2}\right)^{2}+\left(\Delta_{1}-\mathrm{x}\right)^{2}\right]^{-1}\left(\Delta_{1}-\mathrm{x}\right) \mathrm{dx}
\end{aligned}
$$

Donde se ha definido $\mathrm{x}=\xi-\omega_{0}$ y $\Delta_{1}=\omega_{1}-\omega_{0}$. Si expresamos de forma equivalente los términos $\left\langle\mathrm{A}^{(1)}\right\rangle_{\xi}, \ldots,\left\langle\mathrm{A}^{(\mathrm{n})}\right\rangle_{\xi}$, generamos las siguientes relaciones de recurrencia:

$$
\begin{aligned}
& \operatorname{Re}\left\langle\mathrm{A}^{(\mathrm{n})}\right\rangle=\frac{\mathrm{T}_{2}}{2 \mathrm{n}}\left[\mathrm{T}_{2} \operatorname{Re}\left\langle\mathrm{A}^{(\mathrm{n} 1)}\right\rangle \quad \frac{\partial}{\partial\left(1 / \mathrm{T}_{2}\right)} \operatorname{Re}\left\langle\mathrm{A}^{(\mathrm{n} 1)}\right\rangle\right], \\
& \operatorname{Im}\left\langle A^{(\mathrm{n})}\right\rangle=\frac{\mathrm{T}_{2}}{2 \mathrm{n}}\left[\frac{\partial}{\partial\left({ }_{1}\right)} \operatorname{Re}\left\langle\mathrm{A}^{(\mathrm{n} 1)}\right\rangle\right]
\end{aligned}
$$

Los resultados muestran que ahora no sólo podemos relacionar todas las susceptibilidades (incoh, coh, acop) entre sí, sino que además podemos tener todos los términos de cada suma, expresados como función del término de orden cero $\left\langle\mathrm{A}^{(0)}\right\rangle_{\xi}$. Esto permite un ahorro importante de tiempo al evaluar numéricamente las susceptibilidades, así como inferir sobre su interpretación física en relación a los procesos no lineales que tengan lugar. Expresando las ecs. (28) de la forma:

$$
\operatorname{Re}\left\langle\mathrm{A}^{(0)}\right\rangle=\frac{\mathrm{L}}{\mathrm{T}_{2}} \int_{\infty}^{\infty}{ }_{1}(\mathrm{x}){ }_{2}\left(\begin{array}{ll}
\mathrm{I}_{1} & \mathrm{x}
\end{array}\right) \mathrm{dx}
$$




$$
\begin{aligned}
& \operatorname{Im}\left\langle A^{(0)}\right\rangle_{\xi}=-\frac{\sigma_{L}}{T_{2} \pi} \int_{-\infty}^{\infty} \vartheta_{1}(x) \vartheta_{3}\left(\Delta_{1}-x\right) d x
\end{aligned}
$$

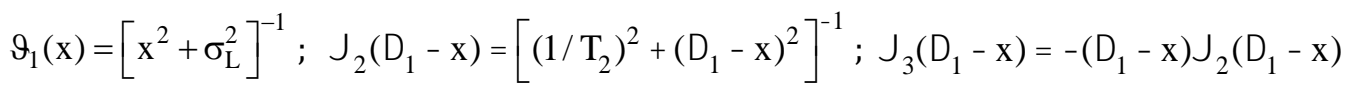

y aplicando el teorema de convolución [12,23], tenemos finalmente:

$$
\begin{aligned}
& \operatorname{Re}\left\langle\mathrm{A}^{(0)}\right\rangle_{\xi}=\frac{\sigma_{\mathrm{L}}+1 / \mathrm{T}_{2}}{\sqrt{2 \pi}\left(\Delta_{1}^{2}+\left(\sigma_{\mathrm{L}}+1 / \mathrm{T}_{2}\right)^{2}\right)} \\
& \operatorname{Im}\left\langle\mathrm{A}^{(0)}\right\rangle_{\xi}=\frac{\Delta_{1}}{\sqrt{2 \pi}\left(\Delta_{1}^{2}+\left(\sigma_{\mathrm{L}}+1 / \mathrm{T}_{2}\right)^{2}\right)}
\end{aligned}
$$

Con las expresiones anteriores, hemos definido completamente todas las magnitudes de interés, que nos permite evaluar las polarizaciones locales macroscópicas no lineales asociadas a cada uno de los campos electromagnéticos. Es necesario, resaltar el hecho que en todos los casos hemos obtenido expresiones analíticas y cerradas las cuales permiten hacer interpretaciones físicas y asociarlas con los procesos multifotónicos que tienen lugar. En las ecs. $(32,33)$ puede observarse que al hacer $\sigma_{\mathrm{L}} \rightarrow 0$ desaparece el efecto del solvente, tal como lo describimos en términos del corrimiento de la frecuencia de Bohr del sistema de dos niveles. En este punto incluimos una notación que identifica casos con efectos del solvente (CES) de aquellos, donde no haya efectos del solvente (SES).

\section{Propagación de los campos electromagnéticos. Intensidad de la señal de Mezcla de cuatro ondas.}

Hasta aquí se ha descrito como se induce la polarización no lineal en un punto de la muestra. En lo sucesivo, abordamos el problema de propagación de los campos electromagnéticos a lo largo del camino óptico del material. Usando la ecuación de Maxwell:

$$
\nabla^{2} \overrightarrow{\mathrm{E}}(\mathrm{r}, \mathrm{t})-\frac{1}{\mathrm{c}^{2}}\left(\frac{\partial^{2} \overrightarrow{\mathrm{E}}(\mathrm{r}, \mathrm{t})}{\partial \mathrm{t}^{2}}\right)=\frac{4 \pi}{\mathrm{c}^{2}}\left(\frac{\partial^{2} \overrightarrow{\mathrm{P}}(\mathrm{r}, \mathrm{t})}{\partial \mathrm{t}^{2}}\right)
$$

Y Considerando la aproximación de la envolvente suave [23], obtenemos el siguiente conjunto de ecuaciones para la propagación de los campos a lo largo del camino óptico z:

$$
\begin{aligned}
& \frac{d \tilde{E}_{1}\left(\omega_{1}, z\right)}{d z}=-\alpha_{1}\left(\omega_{1}, z\right) \tilde{E}_{1}\left(\omega_{1}, z\right) \\
& \frac{d \tilde{E}_{2}\left(\omega_{2}, z\right)}{d z}=-\alpha_{2}\left(\omega_{2}, z\right) \tilde{E}_{2}\left(\omega_{2}, z\right)+\Psi_{2}\left(\omega_{2}, z\right) E_{3}^{*}\left(\omega_{3}, z\right) \exp \left(i \Delta k_{z} z\right) \\
& \frac{d \tilde{E}_{3}\left(\omega_{3}, z\right)}{d z}=-\alpha_{3}\left(\omega_{3}, z\right) \tilde{E}_{3}\left(\omega_{3}, z\right)+\Psi_{3}\left(\omega_{3}, z\right) E_{2}^{*}\left(\omega_{2}, z\right) \exp \left(i \Delta k_{z} z\right)
\end{aligned}
$$

donde $\alpha_{\mathrm{m}}\left(\omega_{\mathrm{m}}, \mathrm{z}\right)$ como el coeficiente de absorción no lineal a la frecuencia $\omega_{\mathrm{m}}$, que está dado por:

$$
{ }_{m}\left({ }_{m}, z\right)=\frac{2 m_{m}\left({ }_{m}, z\right) c}{\operatorname{Im}}{ }_{\text {eff }}\left({ }_{m}, z\right) \quad m=1,2,3 .
$$

Aquí, hemos definido

$$
\chi_{\text {eff }}\left(\omega_{\mathrm{k}}, \mathrm{z}\right)=\left[\chi^{(\mathrm{incoh})}\left(\omega_{\mathrm{k}}, \mathrm{z}\right)+\left(\delta_{\mathrm{k}, 2}+\delta_{\mathrm{k}, 3}\right) \tilde{\chi}^{(\mathrm{coh})}\left(\omega_{\mathrm{k}}, \mathrm{z}\right)\right] \mathrm{k}=1,2,3
$$


$\eta_{\mathrm{k}}\left(\omega_{\mathrm{k}}, \mathrm{z}\right)$ es el índice de refracción no lineal dado por:

$$
{ }_{k}\left({ }_{k}, z\right)=\left[\begin{array}{lll}
2 \\
0
\end{array}+4 \operatorname{Re}_{\text {eff }}\left({ }_{k}, z\right)\right]^{1 / 2}, \quad \eta_{0}=\left[1+4 \pi \operatorname{Re} \chi^{S V}\left(\omega_{k}, z\right)\right]^{1 / 2}
$$

Por otra parte, los factores de acoplamiento están dados por:

$$
\Psi_{j}\left(\omega_{j}, z\right)=\frac{2 i \pi \omega_{j}}{\eta_{j}\left(\omega_{j}, z\right) c} \chi^{(a c o p)}\left(\omega_{j}, z\right) \tilde{E}_{1}\left(\omega_{1}\right) \tilde{E}_{1}\left(\omega_{1}\right) \text { para } j=2,3
$$

En las ecs.(35-37) hemos considerado $\tilde{E}_{j}\left(\omega_{j}, z\right)=\left(E_{0 j} / 2\right) \exp (i \varphi)$ como la envolvente del campo. Finalmente, la componente $\mathrm{z}$ del desajuste del vector de propagación es definido como:

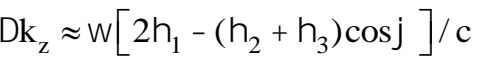

En este punto, es necesario resaltar la ausencia del término $\Psi_{1}\left(\omega_{1}, \mathrm{z}\right)$ en la expresión (35), debido al tratamiento perturbativo a primer orden que se hizo para los haces de prueba y de señal de MCO. Bajo estas condiciones no existen acoplamientos entre los haces que permitan la generación de fotones a la frecuencia del haz de bombeo. Desacoplando las ecs.(36,37) nos queda finalmente:

$$
\frac{\mathrm{d}^{2} \tilde{\mathrm{E}}_{3}(\mathrm{z})}{\mathrm{dz}}+\phi_{1}(\mathrm{z}) \frac{\mathrm{d} \tilde{\mathrm{E}}_{3}(\mathrm{z})}{\mathrm{dz}}+\phi_{2}(\mathrm{z}) \tilde{\mathrm{E}}_{3}(\mathrm{z})=0
$$

donde los coeficientes están dados por:

$$
\begin{aligned}
& \phi_{1}(\mathrm{z})=\alpha_{2}+\alpha_{3}-\left(\frac{\mathrm{d} \ln \Psi_{3}}{\mathrm{dz}}\right)-\mathrm{i} \Delta \mathrm{k}_{\mathrm{z}}-\mathrm{iz}\left(\frac{\mathrm{d} \Delta \mathrm{k}_{\mathrm{z}}}{\mathrm{dz}}\right) \\
& \phi_{2}(\mathrm{z})=\alpha_{2} \alpha_{3}-\Psi_{3} \Psi_{2}^{*}+\left(\frac{\mathrm{d} \alpha_{3}}{\mathrm{dz}}\right)-\alpha_{3}\left[\mathrm{i} \Delta \mathrm{k}_{\mathrm{z}}+\mathrm{iz}\left(\frac{\mathrm{d} \Delta \mathrm{k}_{\mathrm{z}}}{\mathrm{dz}}\right)+\left(\frac{\mathrm{d} \ln \Psi_{3}}{\mathrm{dz}}\right)\right]
\end{aligned}
$$

Es importante resaltar que toda la dependencia en z de los coeficientes de absorción, índices de refracción $\mathrm{y}$ de los factores de acoplamiento, proviene exclusivamente de la dependencia en z que tiene el haz de bombeo, de acuerdo a la ec. (35), la cual podemos proponer que:

$$
\frac{\mathrm{dS}}{\mathrm{dz}}=-2 \alpha_{1} \mathrm{~S}
$$

y por tanto, $\phi_{\mathrm{r}}=\phi_{\mathrm{r}}\left(\mathrm{S}, \frac{\mathrm{dS}}{\mathrm{dz}}\right)(\mathrm{r}=1,2)$. Del análisis de la ec.(43) y de la forma como varían sus coeficientes con la intensidad (saturación) y la derivada con z de este parámetro de intensidad del haz de bombeo, podemos generar las siguientes soluciones de propagación de la señal de MCO. Un primer caso, estudiado inicialmente por Boyd y colaboradores [13], considera que la intensidad del campo de bombeo es estrictamente constante a lo largo del camino óptico. Aquí, los coeficientes $\phi_{1}^{(1)}(\mathrm{z})$ y $\phi_{2}^{(1)}(\mathrm{z})$ están dados por:

$$
\begin{aligned}
& \phi_{1}^{(1)}(\mathrm{z})=2 \alpha_{3}-\mathrm{i} \Delta \mathrm{k}_{\mathrm{z}} \\
& \phi_{2}^{(1)}(\mathrm{z})=\alpha_{3}^{2}-\left|\Psi_{3}\right|^{2}-\mathrm{i} \alpha_{3} \Delta \mathrm{k}_{\mathrm{z}}
\end{aligned}
$$

Con $\mathrm{S}=\mathrm{S}_{0}, \frac{\mathrm{dS}}{\mathrm{dz}}=0$ y por tanto la solución de la ec. (43) para la intensidad normalizada del haz de señal de MCO, es dada por: 


$$
\frac{\mathrm{I}_{3}^{(1)}(\mathrm{z})}{\mathrm{I}_{2}(0)}=\frac{\left|\Psi_{3}(0)\right|^{2} \exp \left[-2 \alpha_{3} \mathrm{z}\right] \operatorname{sen}^{2}\left(\Delta \mathrm{k}_{\mathrm{z}} / 2\right) \mathrm{z}}{(\Delta \mathrm{k})^{2} / 4}
$$

Esta aproximación la notaremos como forma $p$ de propagación en lo que sigue. Desde luego la aproximación $\mathrm{dS} / \mathrm{dz}=0$ no es generalmente válida, por lo que se hace necesario inducir otra forma de propagación de la señal a lo largo del camino óptico (forma q). En esta consideramos que la derivada espacial del parámetro de saturación es pequeña, pero no es despreciable. En este caso la derivada sería proporcional a la saturación, $d S / d z \approx-2 \alpha_{1}^{\left(S_{0}\right)} S_{0}$ donde $S(z) \approx S_{0}$. Consecuentemente, las funciones $\phi_{1}^{(2)}(\mathrm{z})$ y $\phi_{2}^{(2)}(\mathrm{z})$ vuelven a ser aproximadamente constantes y sus valores pueden ser evaluados en el origen. De tal forma que,

$$
\begin{aligned}
& \phi_{1}^{(2)}(\mathrm{z})=2 \alpha_{3}+\left(2 \alpha_{1}^{\left(\mathrm{S}_{0}\right)} \mathrm{S}_{0}\right)\left[\left(\frac{\mathrm{d} \ln \Psi_{3}}{\mathrm{~d} S}\right)+\mathrm{iz}\left(\frac{\mathrm{d} \Delta \mathrm{k}_{\mathrm{z}}}{\mathrm{dS}}\right)\right]-\mathrm{i} \Delta \mathrm{k}_{\mathrm{z}} \\
& \phi_{2}^{(2)}(\mathrm{z})=\alpha_{3}^{2}-\left|\Psi_{3}\right|^{2}-\mathrm{i} \alpha_{3} \Delta \mathrm{k}_{\mathrm{z}}+\left(2 \alpha_{1}^{\left(\mathrm{S}_{0}\right)} \mathrm{S}_{0}\right)\left\{\alpha_{3}\left(\frac{\mathrm{d} \ln \Psi_{3}}{\mathrm{dS}}\right)-\left(\frac{\mathrm{d} \alpha_{3}}{\mathrm{dS}}\right)+\mathrm{iz} \alpha_{3}\left(\frac{\mathrm{d} \Delta \mathrm{k}_{\mathrm{z}}}{\mathrm{dS}}\right)\right\}
\end{aligned}
$$

En este caso, la intensidad normalizada de la señal de MCO es expresada como:

$$
\frac{\mathrm{I}_{3}^{(2)}(\mathrm{z})}{\mathrm{I}_{2}(0)}=\frac{\left|\Psi_{3}\right|^{2}}{\left|\phi_{12}^{(2)}\right|^{2}} \exp \left[-\left(2 \alpha_{3}^{2}+\left(2 \alpha_{1}^{\left(\mathrm{S}_{0}\right)} \mathrm{S}_{0}\right) \frac{\mathrm{d}}{\mathrm{dz}} \ln \left|\Psi_{3}\right|^{2}\right) \mathrm{z}\right] \operatorname{sen}^{2}\left(\operatorname{Im} \phi_{12}^{(2)}\right) \mathrm{z}
$$

donde

$$
\phi_{12}^{(2)}=\frac{1}{2} \sqrt{\left(\phi_{1}^{(2)}\right)^{2}-4 \phi_{2}^{(2)}}=\frac{\mathrm{i}}{2}\left\{\left(\Delta \mathrm{k}_{\mathrm{z}}\right)^{2}-8 \mathrm{~S}_{0} \alpha_{1}^{\left(\mathrm{S}_{0}\right)}\left[\frac{\mathrm{d} \alpha_{3}}{\mathrm{dS}}+\frac{\mathrm{d} \Delta \mathrm{k}_{\mathrm{z}}}{\mathrm{dS}}\right]\right\}^{1 / 2}
$$

Finalmente, analizamos un tercer (3) caso (forma $r$ ) donde la variación de la intensidad del bombeo a través del camino óptico es significativa. En este caso, la saturación del haz de bombeo es de la forma:

$$
\operatorname{Sexp}(4 \mathrm{~S} / \mathrm{b})=\exp \left[2 \mathrm{z}+4 \mathrm{~S}_{0} / \mathrm{b}\right] \mathrm{S}_{0}
$$

donde $\Theta=\frac{2 \pi \omega_{1} \mu_{\mathrm{ab}}^{2} \rho_{\mathrm{D}}^{(0)} \mathrm{T}_{2}}{\mathrm{c} \eta_{1} \hbar} \quad$ y $\quad \mathrm{b}=1+\Delta_{1}^{2} \mathrm{~T}_{2}^{2}$.

La ec.(49) tiene como incógnita el parámetro de saturación S, relacionado con la saturación inicial a través de la función exponencial, por lo que se convierte en una ecuación trascendente, la cual no admite una solución analítica única. En casos extremos aproximados se tiene:

$$
\begin{aligned}
& \mathrm{S}=\exp \left[-2 \Theta z+4 \mathrm{~S}_{0} / \mathrm{b}\right] \mathrm{S}_{0} \\
& \mathrm{~S}=\left(-2 \Theta z \frac{\mathrm{b}}{4}+\mathrm{S}_{0}\right)+\frac{\mathrm{b}}{4} \ln \mathrm{S}_{0}
\end{aligned}
$$

En este trabajo se consideran variaciones de saturación para las cuales $S$ exp $(4 \mathrm{~S} / \mathrm{b})$, por lo que la saturación se escribe de acuerdo a la ec. (50.a). Para esta forma de propagación, la intensidad normalizada de la señal de MCO queda expresada como:

$$
\frac{I_{3}^{(3)}(\mathrm{z})}{\mathrm{I}_{2}(0)}=\frac{\left|\Psi_{3}\right|^{2} \exp \left(-\frac{\phi_{1}^{(3)}(\mathrm{z})}{2} \mathrm{z}\right)[\exp (\mathrm{Az})-\exp (-\mathrm{Az})]}{2 \mathrm{~A}}, \mathrm{~A}=\frac{1}{2}\left[\left(\phi_{1}^{(3)}\right)^{2}-4 \phi_{2}^{(3)}\right]^{1 / 2} .
$$


Es necesario destacar que los dos primeros casos tenemos soluciones analíticas relativamente sencillas, que describen el cambio de la intensidad normalizada de la señal de MCO conforme el camino óptico, mientras que el tercer caso, amerita soluciones numéricas.

\section{Resultados y discusión}

La estrategia de cálculo planteada en este trabajo es válida para cualquier sistema molecular (modelado a través de dos niveles electrónicos), sensible a radiación de alta intensidad; sin embargo, en esta sección hemos seleccionado para el estudio de la propagación de la señal de MCO, los parámetros moleculares (experimentales) del colorante orgánico malaquita verde [24].

\subsection{Estudio de propagación de la señal de MCO}

En esta sección se muestran las tres formas de propagación espacial de la señal de MCO tomando en cuenta diferentes condiciones experimentales, en presencia y ausencia del efecto del solvente. Los resultados obtenidos consideraron los siguientes valores: $\Delta_{1}=11 \mathrm{ps}^{-1}$, longitud óptica, $0 \leq \mathrm{z} \leq 0.04 \mathrm{~cm}$, concentración química $\mathrm{N}$, parámetro de saturación $\mathrm{S}_{0}$, tiempos de relajación (longitudinal y transversal), y varianza de la distribución lorentziana $\sigma_{\mathrm{L}}$.

\subsubsection{Primera forma de propagación para distintos valores de la magnitud del campo eléctrico en presencia y ausencia del baño térmico}

Los perfiles de intensidad con respecto a variaciones de la longitud óptica y de la magnitud del campo aplicado, permiten ver a modo general, la dependencia de las formas de propagación con el campo eléctrico. Sin embargo, para algunas formas de propagación, hay un valor de la saturación a cada valor de z, por tanto, aquí sólo se muestran las superficies de intensidad del primer modo de propagación en presencia (CES) y ausencia del solvente (SES). En la Fig. 1 se muestran las superficies de intensidad obtenidas bajo las siguientes condiciones: $\mathrm{N}=1.0 \mu \mathrm{M}, \mathrm{T}_{1}=10 \mathrm{~T}_{2}$ para $\mathrm{T}_{2}=1.1 \mathrm{ps}$.
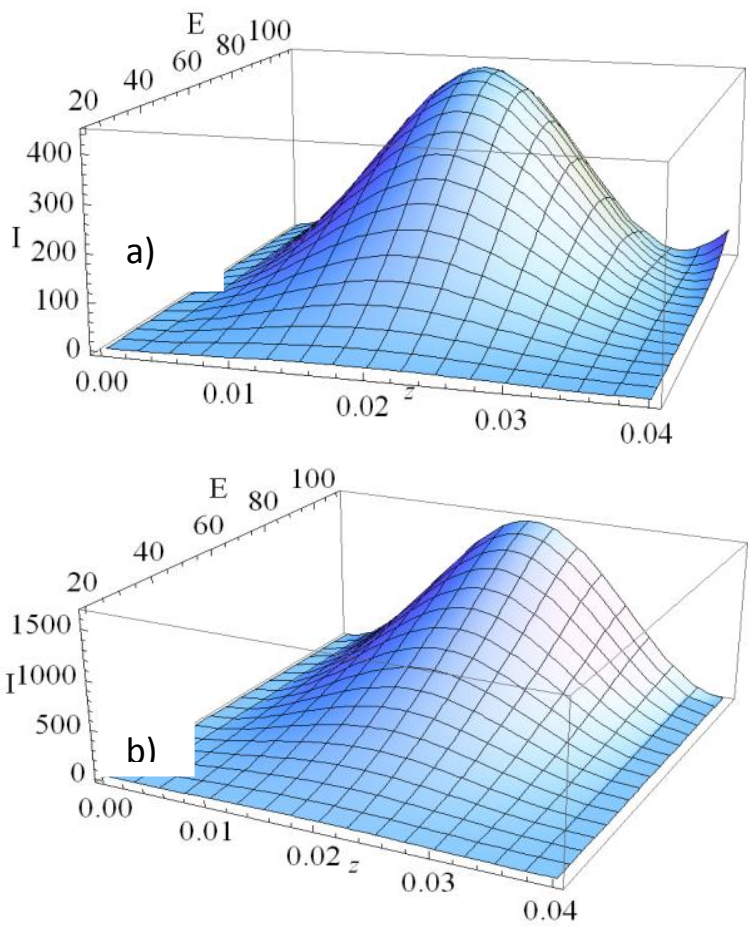

Fig. 1. Dependencia del primer modo de propagación espacial de la señal de MCO con el campo eléctrico. a) CES b) SES

\subsubsection{Formas de propagación para distintos valores de saturación en presencia y ausencia del} baño térmico

En esta sección se presenta un análisis del efecto de la saturación inicial sobre los tres modos de propagación indicados arriba, en presencia (CES) y ausencia del baño térmico (SES) en cada caso. 

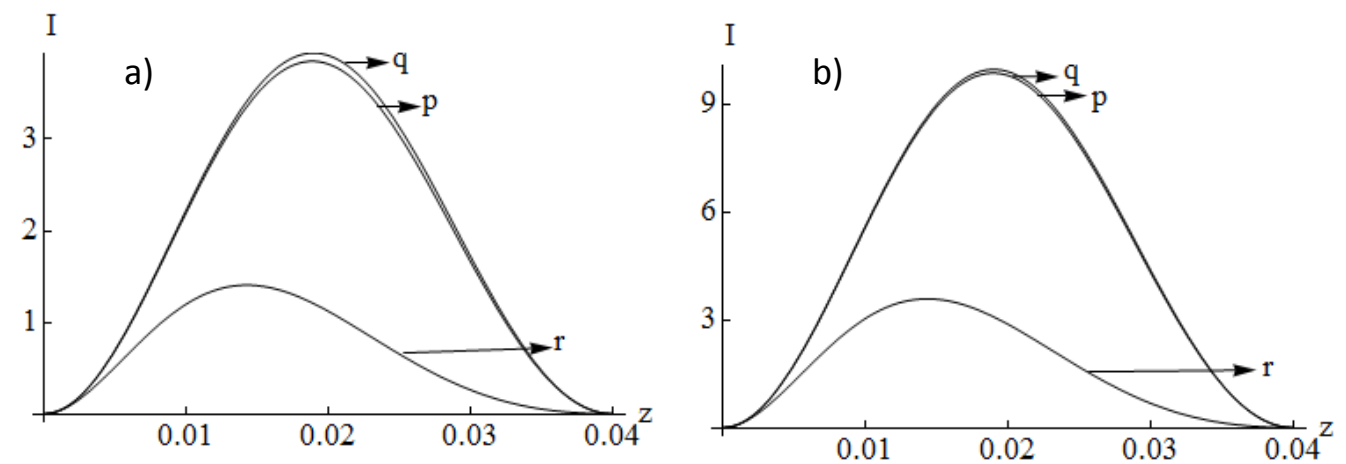

Fig. 2. Propagación de la señal de MCO para $\mathrm{S}_{0}=0.02$ y $\mathrm{N}=1.0 \mathrm{M}$ a) CES b) SES. Formas de propagación: p: primera, q: segunda, r: tercera.

En la Fig. 2 se superponen los perfiles de intensidad de la señal de MCO para un intervalo de longitud óptica dado por $0 \leq \mathrm{z} \leq 0.04 \mathrm{~cm}$, con una relación de tiempos de relajación $\mathrm{T}_{1}=10 \mathrm{~T}_{2}$ con $\mathrm{T}_{2}=1.1 \mathrm{ps}$. Se aprecian perfiles similares, aún cuando las intensidades de propagación del sistema en ausencia del efecto del reservorio térmico son mayores. En general, se observa que la intensidad de la propagación de la señal de MCO se incrementa, según las distintas formas, en el siguiente orden: $r \ll p \approx q$.

Analizando las tres formas de propagación, se deduce que en un límite de aproximación, el primer modo asume que durante el recorrido óptico la intensidad del campo incidente es constante; por su parte, en el segundo modo de propagación, se considera la variación espacial de la saturación, no obstante, la saturación puede fijarse al valor inicial. Finalmente en el tercer nivel de aproximación, la saturación se evalúa como un parámetro independiente del valor inicial que varía a través del recorrido óptico. Por tanto, para estas condiciones experimentales, las formas de propagación primera y segunda, son equivalentes, representando éstas, frente al tercer modo, una subestimación de la intensidad del campo de bombeo. Referente al sistema en ausencia del efecto del solvente, se observa que las respuestas para los tres modos de propagación son más intensas que para el sistema que interactúa con el reservorio térmico y al igual que éste, las formas de propagación primera y segunda se imponen sobre la otra aproximación. El hecho que las intensidades de la señal en ausencia del efecto del solvente sean mayores que para el caso de interacción solvente-soluto, está directamente relacionado con las respuestas ópticas, dado que, tanto el índice de refracción como el coeficiente de absorción no lineal, son más intensos en el caso de interacción nula soluto-solvente.

Análogamente, se calcularon las intensidades de propagación para la señal de MCO, tanto CES y SES, para un valor de saturación $S_{0}=0.2$. Estas curvas se presentan en la Figura 3 .
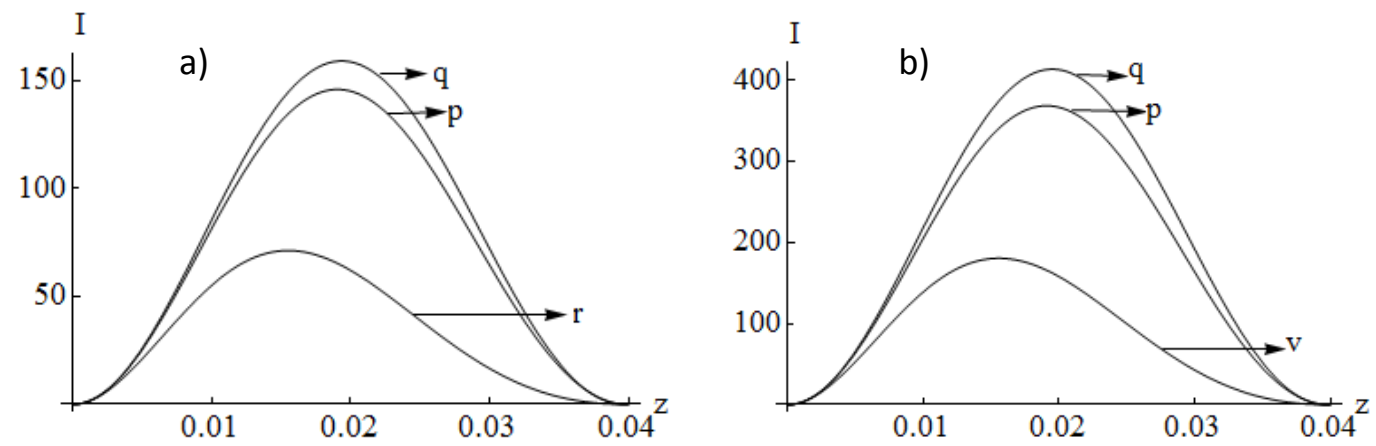

Fig. 3. Propagación de la señal de MCO para $\mathrm{S}_{0}=0.2$ y N=1.0 M a) CES b) SES. Las formas de propagación $p, q$, y $r$ son las que se indican en el texto.

Aquí puede observarse que para esas condiciones experimentales, la primera $(p)$ y segunda forma $(q)$ de propagación, de ambos sistemas (en presencia del efecto del baño térmico y en ausencia de él) tienen intensidades superiores a la tercera forma $(r)$ de propagación. Sin embargo, a diferencia del caso mostrado en la Fig. 2, los modos de propagación primero y segundo no son equivalentes dado que tienen 
un porcentaje de error superior a la tolerancia permitida (10\%). Análogo al sistema con saturación inicial $\mathrm{S}_{0}=0.02$, estas formas de propagación con respecto a la tercera aproximación representan una sobreestimación de la intensidad de la fuente, con lo que se obtienen estas elevadas respuestas. Asimismo, bajo las condiciones experimentales empleadas en los casos anteriores, se obtuvieron las intensidades de propagación de la señal de MCO para un valor de saturación $S_{0}=0.4$. Tales intensidades se presentan en la Fig. 4, donde se observan las respuestas en presencia y ausencia del efecto del solvente.
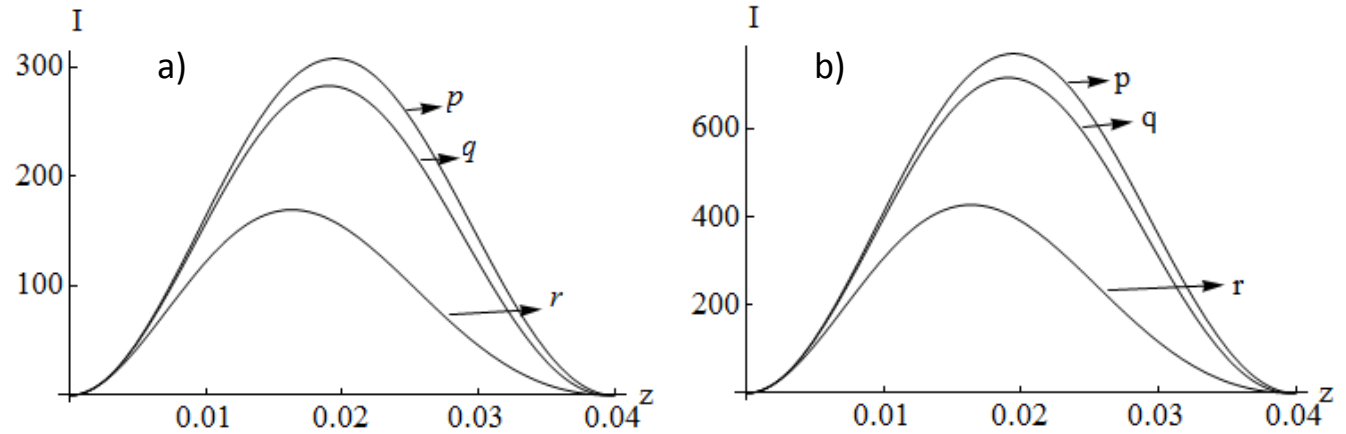

Fig. 4. Propagación de la señal de $\mathrm{MCO}$ para $\mathrm{S}_{0}=0.4, \mathrm{~N}=1.0 \mathrm{M}$ a) CES b) SES. Las formas de propagación $p, q$, $\mathrm{y} r$ son las que se indican en el texto.

La Fig. 4 muestra las intensidades de propagación obtenidas para el doble del valor de la saturación de los resultados reportados en la figura anterior. Si bien se observan las mismas tendencias, tanto en presencia como ausencia del efecto del solvente, el efecto de la saturación en la intensidad de las respuestas no es proporcional. Pudiera considerarse que dado que se incrementa el doble de la saturación con respecto a la Fig. 3 entonces los máximos de las curvas duplican exactamente el máximo de las intensidades obtenidas a $\mathrm{S}_{0}=0.2$, sin embargo, no es así. Particularmente, la segunda forma de propagación para valores de $\mathrm{S}_{0}=0.4$, presenta un incremento mayor al $100 \%$ frente a la misma aproximación para $\mathrm{S}_{0}=0.2$.

\subsection{Equivalencia de los modos de propagación en presencia y ausencia del baño térmico}

En la Fig. 5 hemos querido mostrar bajo cuales condiciones las dos primeras formas de propagación puedan sustituir el tercer modo, que consideramos más exacto. Para ello, hemos definido un límite de tolerancia del $10 \%$ sobre la tercera forma de propagar, y evaluamos bajo distintas condiciones la intensidad de la señal de MCO. Este porcentaje de error relativo, permite con un límite de confianza del $90 \%$, identificar, bajo cuáles condiciones de los parámetros seleccionados, es posible emplear una aproximación más simple (primer o segundo modo) para describir la propagación de campos, para la cual los resultados sean comparables a los derivados de la aproximación numérica.

En general, se observa que la concentración química es determinante en la equivalencia de los modos de propagación. A concentraciones del orden micromolar, sólo puede aplicarse el tercer nivel. Cuando la saturación inicial es $\mathrm{S}_{0}=0.4$, las formas de propagación primera y segunda son equivalentes entre sí, sin embargo, frente a la tercera forma de propagación, representan una sobreestimación del campo incidente. Cuando la saturación inicial es $S_{0}=1$, la intensidad del campo eléctrico incidente en la primera y segunda forma de propagación, se sobreestima y subestima, respectivamente. Por su parte, cuando las concentraciones son inferiores a la escala micromolar, los tres modos de propagación son equivalentes, y todos los niveles de aproximación se superponen. Bajo estas condiciones, independientemente de la magnitud del campo eléctrico aplicado, y por ende de la saturación, la propagación espacial de campos se puede estudiar empleando la aproximación analítica (primera o segunda forma de propagación) en lugar de la numérica. Para estos casos, los cálculos se reducen considerablemente dado que las expresiones de intensidad son más sencillas 

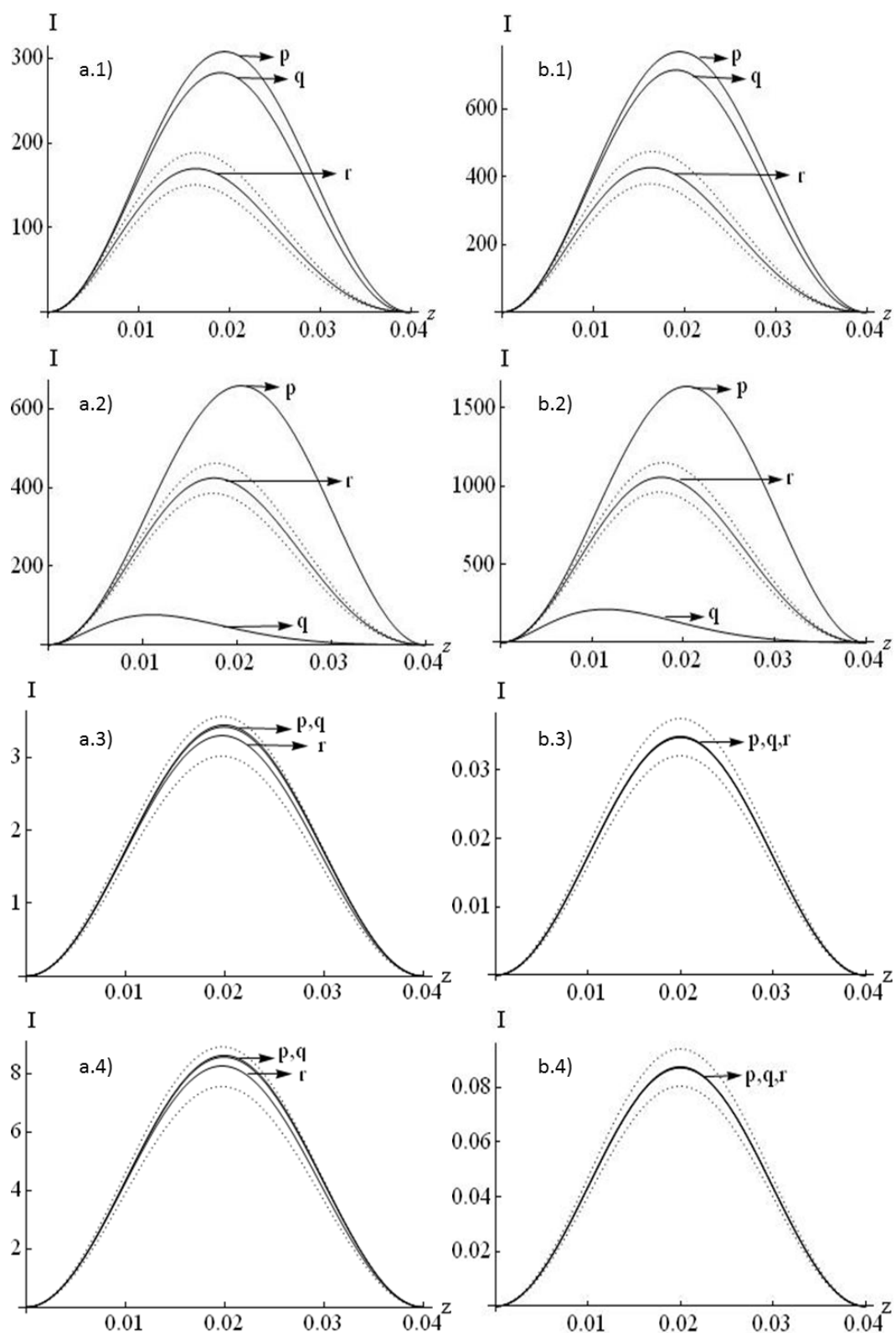

Fig. 5. Propagación de la señal de MCO. a) CES b) SES 1) $\mathrm{S}_{0}=0.4, \mathrm{~N}=1.0 \mathrm{M}$

2) $\left.\left.\mathrm{S}_{0}=1.0, \mathrm{~N}=1.0 \mathrm{M}, 3\right) \mathrm{S}_{0}=0.4, \mathrm{~N}=0.1 \mathrm{M}, 4\right) \mathrm{S}_{0}=0.4, \mathrm{~N}=0.01 \mathrm{M}$. Las formas de propagación $p, q$, y $r$ son las que se indican en el texto. Tolerancia $\pm 10 \%$ alrededor de $r$.

\subsection{Efecto de la concentración sobre las intensidades de propagación}

En las secciones anteriores se ha visto que el valor inicial de la saturación influye sobre la intensidad de la propagación espacial de la señal de MCO. Sin embargo, existen otros factores que afectan la intensidad de las respuestas como, por ejemplo, la concentración de la solución química. En este sentido, experimentalmente se considera que una elevada densidad de soluto no permite el paso óptico, es preciso trabajar con concentraciones molares diluidas (del orden micromolar) que admitan la propagación de campos en el medio material [13]. Adicionalmente, si se trabaja con concentraciones $0.1 \mu \mathrm{M}$, las tres formas de propagación son equivalentes, por tanto en esta sección se presenta el efecto de la concentración, en un rango de $0.1 \mu \mathrm{M}$, sobre los modos de propagación. Específicamente seleccionamos los valores de concentración $\mathrm{N}=0.1 \mu \mathrm{M}, 0.09 \mu \mathrm{M}$ y $0.08 \mu \mathrm{M}$. En la Fig. 6 se muestran los resultados obtenidos considerando un valor fijo de la saturación inicial $S_{0}=0.4$, con una longitud óptica $0 \leq z \leq 0.04$ 
cm y $\Delta_{1}=11 \mathrm{ps}^{-1}$, con una relación de tiempos de relajación $\mathrm{T}_{1}=10 \mathrm{~T}_{2}$, donde $\mathrm{T}_{2}=1.1 \mathrm{ps}$, con un valor fijo de la varianza de la distribución lorentziana $\sigma_{\mathrm{L}}=\mathrm{T}_{1}^{-1} \mathrm{~s}^{-1}$.
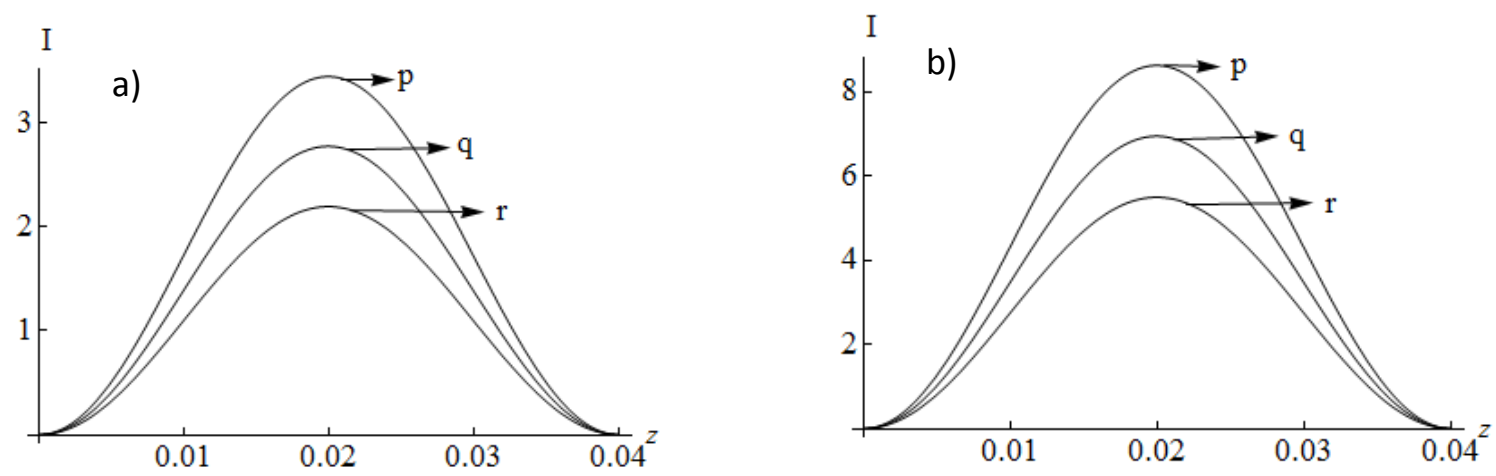

Fig. 6 Intensidad de la señal de MCO en sus distintas formas de propagación, $S_{0}=0.4$ a) CES. b) SES . Aquí la notación es $\mathrm{r}: 0.08 \mathrm{M}, \mathrm{q}: 0.09 \mathrm{M}, \mathrm{p}: 0.1 \mathrm{M}$.

A partir de la Fig. 6 se observa que en ambos casos $(a, b)$, las intensidades de propagación dependen linealmente de la concentración. Naturalmente, al aumentar la dilución del sistema se favorece la absorción del campo eléctrico que pasa a través del medio material; sin embargo, concentraciones muy bajas (inferiores a la escala nanomolar) pudieran no representar adecuadamente el conjunto de partículas que, debido a colisiones, obedece a una distribución de probabilidad específica.

\subsection{Efecto de los tiempos de relajación sobre las intensidades de propagación en presencia del baño térmico}

Al igual que en las propiedades ópticas, los tiempos de relajación, longitudinal y transversal, tienen un efecto sobre las intensidades de propagación. Por tanto, en esta sección se muestran tales efectos sobre el sistema que interactúa con el reservorio térmico, considerando una concentración $0.1 \mu \mathrm{M}$ para la cual las formas de propagación son equivalentes.

De la Fig. 7 se infiere que las intensidades de propagación son sensibles a las distintas relaciones y magnitudes de tiempos de relajación estudiados. Puede observarse que para cada relación, existe un orden de magnitud en la intensidad, por lo cual las curvas se presentan por separado. Cuando el tiempo de relajación longitudinal es mayor que el tiempo de relajación transversal, la intensidad de los modos de propagación es menor que en el caso contrario, aunque mayor al caso en el que los tiempos de relajación son iguales. Con respecto a la relación $T_{1}<T_{2}$, para un valor constante de $T_{2}$, cuanto menor es $T_{1}$, menor es la intensidad de propagación. Por lo general, la componente de relajación en dirección del campo, $\mathrm{T}_{1}$ es mayor a la componente de relajación transversal, $\mathrm{T}_{2}$, esto debido al decaimiento exponencial asociado a los procesos $\mathrm{T}_{2}$. Así, el tiempo requerido para que el sistema después de una excitación retome el equilibrio es menor a $\mathrm{T}_{1}$. Por lo cual, la mayoría de los sistemas moleculares expuestos a campos electromagnéticos responden a relación $\mathrm{T}_{1}>\mathrm{T}_{2}$. 

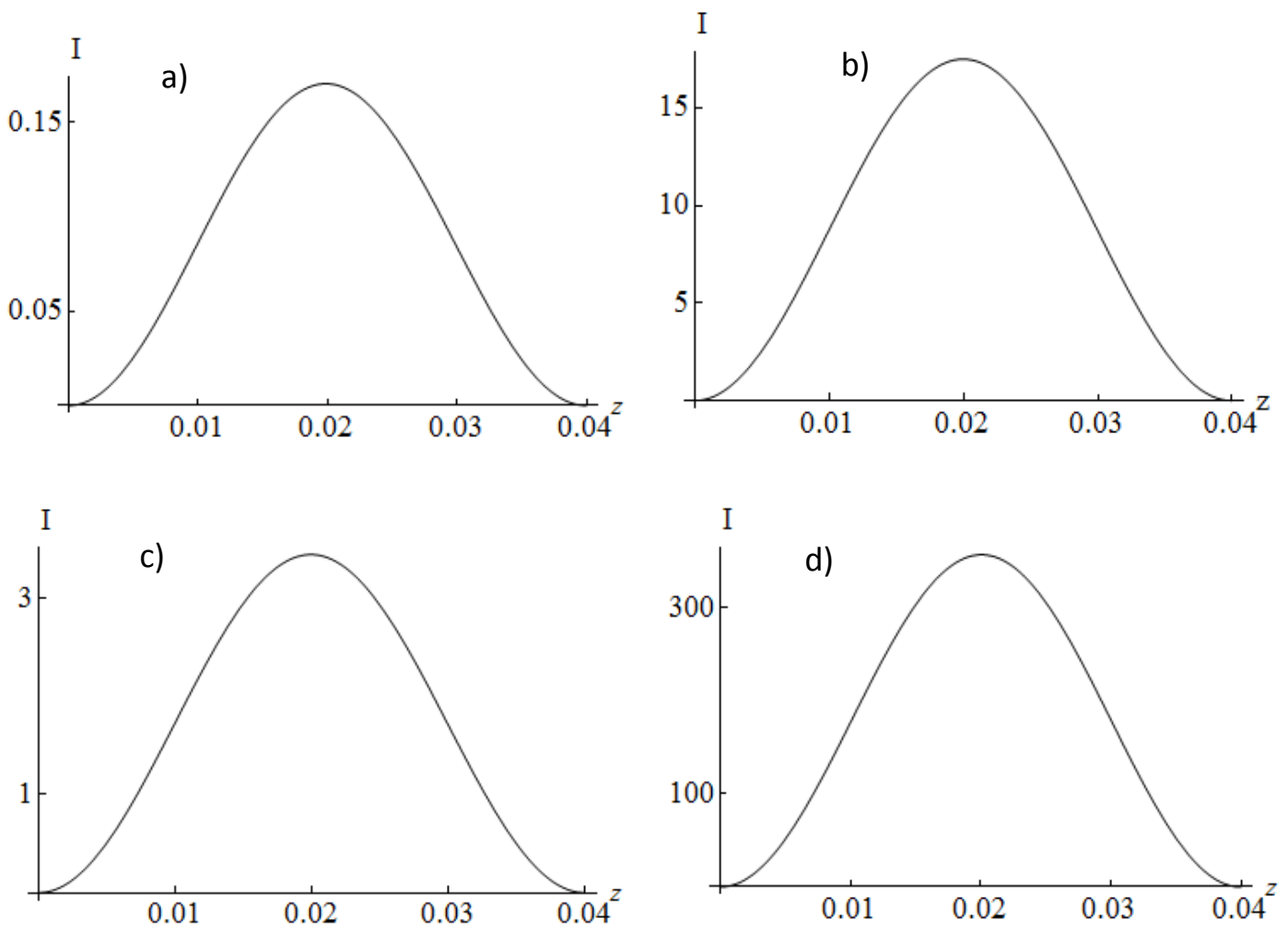

Fig. 7. Equivalencia de los modos de propagación de la señal de MCO $\mathrm{S}_{0}=0.4 ; 0 \quad \mathrm{Z} \quad 0.04 \mathrm{~cm} ; \quad{ }_{1}=11 \mathrm{ps}{ }^{1} ; \quad \mathrm{L}_{2}=\mathrm{T}_{1}{ }^{1} \mathrm{~S}^{1}$ para distintas relaciones de tiempos de relajación a) $\mathrm{T}_{1}=\mathrm{T}_{2}=1.1 \mathrm{ps}$; b) $\mathrm{T}_{1} / \mathrm{T}_{2}=10, \mathrm{~T}_{2}=1.1 \mathrm{ps} ;$ c) $\mathrm{T}_{1} / \mathrm{T}_{2}=0.01, \mathrm{~T}_{2}=11 \mathrm{ps} ;$ d) $\mathrm{T}_{1} / \mathrm{T}_{2}=0.1$

$$
\mathrm{T}_{2}=11 \mathrm{ps}
$$

\subsection{Efecto de la varianza de la distribución lorentziana sobre los modos de propagación}

Como parte de este trabajo, se estudió el efecto de la varianza sobre las propiedades ópticas, lo que demostró una atenuación de las intensidades conforme al incremento de ésta. Sin embargo, para los valores experimentales de tiempos de relajación y varianza de la malaquita verde, las curvas de propagación no presentan tal dependencia, esto debido a la relación entre el tiempo de relajación longitudinal con la varianza. En la Fig. 8, se observa el efecto de la varianza de la distribución sobre un sistema molecular genérico, cuyos tiempos de relajación longitudinal y transversal, son iguales.

El incremento de la varianza en los modos de propagación hace que las intensidades disminuyan, esto debido, principalmente, al decaimiento exponencial de la intensidad, la cual depende del índice de refracción y el coeficiente de absorción.

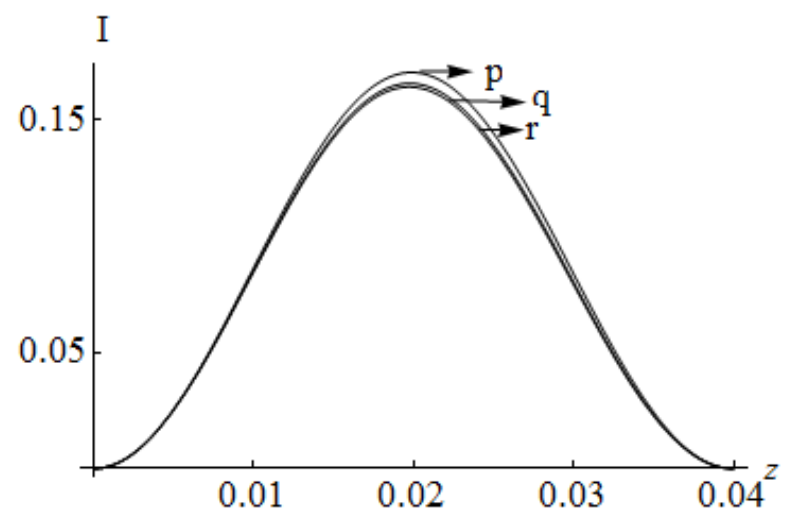

Fig. 8. Propagación de la señal de MCO para $\mathrm{S}_{0}=0.4, \mathrm{~T}_{1}=\mathrm{T}_{2}=1.1 \mathrm{ps} \quad \mathrm{N}=0.1 \mathrm{M}$

Aquí la notación es $\mathrm{p} \mathrm{L}_{\mathrm{L}}=\mathrm{T}_{1}{ }^{1} \mathrm{~s}^{1}, \mathrm{q}:_{\mathrm{L}}=1.5 \mathrm{~T}_{1}{ }^{1} \mathrm{~s}^{1}, \mathrm{r}:_{\mathrm{L}}=2 \mathrm{~T}_{1}{ }^{1} \mathrm{~s}^{1}$ 


\section{Comentarios finales.}

El modelo presentado aquí ha permitido el estudio de los efectos del solvente sobre la manera como se propaga la señal de MCO a lo largo del camino óptico, apreciando cuan sensible con relación a parámetros como la concentración química, tiempos de relajación, saturación del bombeo, varianza de la distribución de probabilidades. Hemos considerado en nuestros cálculos, los parámetros que caracterizan al colorante orgánico malaquita verde. El formalismo aplicado es aplicado en aquellos casos donde es válida la relación $4 \mathrm{~S} / \mathrm{T}_{2}^{2}|\mathrm{D}|^{2} \quad$ 1. Se dedujo que el primer modo de propagación descrito en el texto representa una sobreestimación de la intensidad del campo eléctrico incidente, y que este modo puede aplicarse con cierto éxito para sistemas con saturaciones iniciales elevadas. Se demostró que la segunda forma de propagación discutida permite describir una atenuación de las repuestas obtenidas, principalmente para valores extremos de la saturación. Finalmente, en un tercer modo de propagación, al igual que en el primer modo, se sobreestima la intensidad del campo incidente, excepto para valores elevados de saturación $\left(\mathrm{S}_{0}=1.0\right)$. Observamos por otro parte, que las intensidades de propagación son proporcionales a la concentración e inversamente proporcional a la varianza de la distribución lorentziana empleada. Del conjunto de parámetros empleados, la concentración química de la solución resultó ser más determinante en la equivalencia de los modos de propagación, específicamente, a valores menores de las escalas micromolares.

\section{Agradecimientos}

JLP y LGR agradecen a la Secretaría Nacional de Ciencia, Tecnología e Innovación (SENESCYT) por el financiamiento brindado a través del Proyecto Becas Prometeo 